\title{
Assessing the Role of Machine Learning in Robotics
}

\author{
Santhosh S, Karthik J, Chesmi B R, Anju Thomas, Davis Patel
}

\begin{abstract}
Machine learning is concerned with algorithms inspired by the structure and function of the brain called artificial neural networks. Neural framework offers wide support for machine learning algorithms. It is an interface, library or tool which allows developers to build machine learning models easily, without getting into the depth of the underlying algorithms. The neural framework is an exceptionally intricate piece of a person that co-ordinate its activities Moreover, tactile data by transmitting signs to and from various pieces of the body. Neural frameworks are applied to perform object gathering and a grasp orchestrating task. Machine Learning techniques have been applied to many sub problems in robot perception - pattern recognition and self-organisation. Modern robot framework which demands a complete detail of each movement of the robot, which breaks the pick-and-spot issue into about free, computationally conceivable sub-issues as a phase toward a comprehensive endeavour level framework
\end{abstract}

Keywords: Robot, Machine Learning, Pick and Spot, Artificial Intelligence, Framework.

\section{INTRODUCTION}

Artificial Intelligence has permitted a change in outlook in design acknowledgment, from utilizing handmade highlights Moreover, specialized upgrades such as the development of computational force and preparing dataset with measurable classifiers to utilizing universally useful learning systems for learning information-driven portrayals, highlights, and classifiers together. The utilization of the AI world view has encouraged tending to a few PC vision issues more effectively than with conventional methodologies. Actually, in a few PC vision benchmarks, for example, the ones tending to picture order, object identification and acknowledgment, semantic division, and activity acknowledgment, to give some examples, the more significant part of the severe strategies are currently founded on the utilization of profound learning systems. It has just pulled in consideration of the robot vision network. New strategies and calculations are generally evolved inside the PC vision network and afterward moved to the robot vision network.

Revised Manuscript Received on March 05, 2020.

* Correspondence Author

Prof Santhosh S, B.E, MTECH in field of digital electronics and communication.Research Work on Embedded Systems and Robotics.

Karthik J, 4th Year, B.E (Electronics and Communication Engineering) Finalists at eYRC-2018.

Chesmi B R, 4th Year, B.E (Electronics and Communication Engineering) Finalists at eYRC-2018.

Anju Thomas, 4th Year, B.E (Electronics and Communication Engineering) Finalists at eYRC-2018.

Davis Patel, 4th Year, B.E (Electronics and Communication Engineering) Finalists at eYRC-2018.

(C) The Authors. Published by Blue Eyes Intelligence Engineering and Sciences Publication (BEIESP). This is an open access article under the CC BY-NC-ND license (http://creativecommons.org/licenses/by-nc-nd/4.0/)

\section{LITERATURE SURVEY}

Feng and Hoberock et al. [1] exhibited a neural system sway to deal with tackling the dynamic booking issue for pick-place tasks of a robot-vision-following framework.

An ideal booking issue is planning to limit robot preparation time without requirement infringement.

For gathering each item, this booking is an ongoing enhancement issue that should discuss again. A plan which utilizes neural systems to take in the mapping from object design space to ideal request space is disconnected and to review online what it has discovered is introduced. The thought was executed in a genuine framework to take care of an issue in enormous business dishwashing tasks. Trial results have indicated that with four distinct articles, time reserve funds of up to $21 \%$ are conceivable over first-come, first-served plots right now utilized in industry.

Nagchaudhuri et al. [2] portrayed an instructive task utilizing a modern robot and a coordinated vision framework. The undertaking exhibits adaptability given by a coordinated vision framework in an automated work-cell. Robots and numerically controlled machines permit simple new capacity to adjust different tasks, which is predefined and subsequently discovers widespread use of assembling robotic automation in plants. Albeit programmable robots have given some level of adaptability in mechanization endeavours. To a great extent, they joined in assembling cells where a portion of the underlying container taking care should assist in inflexible apparatuses. Vision and material detecting ability have bitten by bit being fused in mechanical work-cells in the advanced industrial facilities to give extra adaptability to parts dealing within pick and spot, welding, painting, cleaning, and get together tasks.

Vandenplas et al. [3] have proposed a work that centres around a recently assembled look into a robot. The use of Perception Architecture can recognize round items that will haphazardly dissipate on a table. At that point, utilizing its gripper procedures, they are picked and set inside a bushel the control framework tunes to such extent that the progression of activities runs quickly and safely. Exercises scholarly can spare time for different gatherings keen on building model robots.

Brudka et al. [4] introduced a shrewd robot control framework that utilizes low-quality high estimations to perform high-accuracy acknowledgment and getting a handle on assignments. The framework adaptively reestablishes the ultrasonic picture utilizing approximators dependent on neural systems. Neural systems are likewise applied to perform object grouping and a grip arranging task. Since grip arranging is not impressive, he built up a novel learning plan that utilizes the desire boost approach.

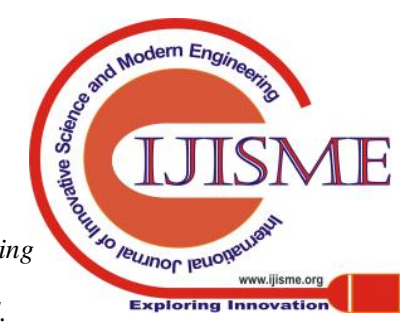


The subsequent framework works precisely and dependably. The fundamental approach can stretch out to other lowquality information issues.

Read and Merwe et al. [5] examined on customary robot-instruct strategies which require cautious and tedious gradual developments when picking and putting smaller scale segments. A quite absolute power should set up. There is an expanding requirement for miniaturized scale material dealing with frameworks that are exact as well as natural. It is relevant in considering a smaller scale piezo fired pick and spot contextual analysis. For the event that this particular power will surpass, it may result in harm. It is particularly important considering the utilization of a Van der Waals gripper for piezo-artistic smaller scale part situating.

Various issues emerge in a progression of activities learned by the framework that can be applied over a coarse $500 \mathrm{~mm}$ run with an excellent three $\mu \mathrm{m}$ repeatability. The framework's capacity to actualize adaptable, responsive and natural control is central to its appropriateness to the considered contextual analysis.

Santana and Cavalcanti et al. [6] exhibited the issue of encouraging an enunciated robot to pick and spot tasks. AI systems are utilized with the Learning by Demonstration (LpD) method to solve this type of issue. Right now, approach the robot follows to figure out how to play out an errand from activities performed by a demonstrator specialist, an individual, or a robot. There are numerous approaches to exhibit conduct for a robot, for instance, by utilizing a succession of caught sensor information on the demonstrator specialist or a video with demonstrator operators. The outcomes that resulted from shows that the proposed arrangement had the option to learn in two situations: information of developments of the end effector in line and information of end effector acknowledging developments in a plane. This work examines the utilization of a learning machine and PC vision for the control of the developments of a mechanical arm.

Andersen et al. [7] proposed a work on a Flexible SkillBased Approach to Recognize Objects in Industrial Scenarios. Conventional mechanical robots are profoundly productive and exact, and along these lines appropriate for completing straightforward, dull undertakings. They are muddled and tedious in the arrangement and to re-program to perform new assignments. Programming endeavours can decrease each essential time, which is only the requirement for a unique body of workers to set up present-day cooperative robots. This paper proposes an ability for acknowledgment and order of various items. The aptitude is parameterized utilizing manual education and AI dependent on SIFT highlights, Bag of Words, and SVM is ut to arrange objects. A client study with 20 test members shows that apply autonomy learners after just a short acquaintance is capable of educating the ability and join it with different aptitudes (pick and spot) to program a total undertaking.

Jones et al. [8] exhibited work on the Handey framework, which breaks the pick-and-spot issue into about free, computationally plausible sub-issues as a stage toward an exhaustive undertaking level framework. An assignment level robot framework is one with undertaking level objectives, for example, "Handle section A and place it inside box B." This sort of determination stands out pointedly from that required for existing modern robot frameworks, which demand a complete detail of each movement of the robot and not just a portrayal of an ideal objective. A significant quality of errand level details is that they are autonomous of the robot playing out the undertaking, though a movement particular is married to a particular robot.

Noble et al. [9] developed work on a remote/-controlled mobile robot and describe software used to generate a data set for vision-based, supervised machine learning applications. He has also demonstrated the use of a generated data set for training a convolutional neural network to detect objects. In his work, he has developed a remote-controlled mobile robot and software, which can record its point of view and generate a data set of training and validation features and labels. In this paper, he described the developed platform, intending to enable other researchers to get started with developing their mobile, machine learning robots, and applications.

Huang et al. [10] talked about on structure and execution of a product framework that is a stage in propelling the innovation toward full robotization at sensible expenses. His framework incorporates much cutting-edge systems in PC vision, profound learning, direction improvement, visual serving to make a library of aptitudes that will play out an assortment of mechanical errands. He exhibited the ability of our framework for performing independent pick-and spot undertakings with a usage utilizing Hoppy robot, which is a mechanical quality robot for industrial facility computerization. He has manufactured a library of programming abilities that utilize to create automated assignments for different pick-and-spot applications. The rest work he has introduced is robot control philosophy, including object identification, acknowledgment, and area of-intrigue, 3D object model remaking, and target $6 \mathrm{D}$ objects present estimation.

\section{CONCLUSION}

The neural system approach is utilized to take care of the dynamic planning issue for pick place activities of a robot-vision neural framework. The robot first is instructed to recognize the item to be picked from encompassing utilizing a preparation picture of the objects. Machine learning has increased understanding of the nature of intelligence and provided an impressive array of application in a wide range of areas. It has honed comprehension of human thinking, and of the idea of insight all in all. Robust perception is a major bottleneck in recognition of people, places, and objects recognizing and predicting human behaviour, goals and intentions.

\section{REFERENCES}

1. K. Feng; L.L. Hoberock," An optimal scheduling of pick place operations of a robot-vision-tracking system by using backpropagation and Hamming networks," Proceedings1992 IEEE International Conference on Robotics and Automation, 1992.

2. A. Nagchaudhuri, "Robotics and machine vision for introduction to flexible automation to engineering undergraduates," 32nd Annual Frontiers in Education, Vol. 1, Nov -2002.

3. S. Vandenplas, "A fast pick-and-place prototype robot: design and control," IEEE Conference on Control Applications (CCA), 2016. 
4. M. Brudka, "Intelligent robot control using ultrasonic measurements," IEEE Transactions on Instrumentation and Measurement, Volume: 51, Jun 2002.

5. S. Read, A. van der Merwe, S. Matope, and A. Smit, "An Intuitive Teachable Micro Material Handling Robot with Van der Waals Gripper," IEEE Conference Handling for Micro- Manufacturing, 2012, chap. 18, pp. 298-314.

6. Orivaldo Santana, Samuel Cavalcanti, "Self- Learning in the Inverse Kinematics of Robotic Arm," IEEE Conf. of Robotics and Autonomous Systems, 57/5, May 2017.

7. Rasmus S. Anderson, "Using Flexible Skill- Based to Recognize Objects in Industrial Scenarios," 47st International Symposium on Robotics, 2016.

8. J.L Jones, "Task-level planning of pick- and - place robot motions," Vol 22, 1989.

9. Frazer K. Noble, "A Mobile Robot Platform for Supervised Machine Learning Applications," 24th International Conference on Mechatronics and Machine Vision in Practice (M2VIP), 2017.

10. Pei-Chi Huang, "A Case Study of Cyber- Physical System Design: Autonomous Pick and Place Robot," IEEE 24th International Conference on Embedded and Real-Time Computing Systems and Applications (RTCSA), 2018

11. Marius Sumanas, Vytautas Bucinskas, Inga Morkvenaite, Andrius Dzedziskis, Tadas Lenkutis, "Implementation of Machine Learning Algorithms for Autonomous Robot Trajectory Resolving," IEEE, 2016.

12. Pariwat Ongsulee, "Artificial Intelligence, Machine Learning, and Deep Learning," Fifteenth International Conference on ICT and Knowledge Engineering, 2017.

13. Nadia Jmour, Sehla Zayen, Afef Abdelkrim, "Convolutional Neural Networks for image classification," IEEE, 2015

14. Nicolas Bredeche, Zhongzhi Shi, "Perceptual Learning and Abstraction in Machine Learning: An Application to Autonomous Robotics," IEEE TRANSACTIONS ON SYSTEMS, MAN, AND CYBERNETICS, 36/2, March 2006.

15. Raunak Sethiya, Mayur Jori, Omkar Mokashi, Taresh Tak, D.P. Salapurkar, "Intelligent Robotic Arm to Pick and Place Target Object," International Research Journal of Engineering and Technology (IRJET), 5/ 4, April- 2018.

16. HAN Jian-Ning, WANG Ming-Quan, "Research on Digital Image Recognition System Based on Multiple Invariant Moments Theory and BP Neural Network," 2nd International Asia Conference on Informatics in Control, Automation and Robotics, 2010.

17. Jinqiang Bai, Shiguo Lian, "Deep Learning- Based Robot for Automatically Picking up Garbage on the Grass," IEEE, 2016.

18. Shuuji Kajita, Kensuke Harada, Hajime Saito, Mitsuharu Morisawa, "A Humanoid Robot Carrying a Heavy Object," IEEE International Conference on Robotics and Automation Barcelona, April 2005.

19. Pratiksha Andhare, Sayali Rawat, "Pick and Place Industrial Robot Controller with Computer Vision," International Journal of Engineering and Technology (IJET), ISSN: 0975-4024, 5/1 Feb-Mar 2013.

20. Robert Platt, Marcus Gualtieri, Andreas ten Pas, "Pick and Place Without Geometric Object Models," IEEE International Conference on Robotics and Automation (ICRA), May 21-25, 2018.

21. Kyekyung Kim, Jaemin Cho, "Dynamic Object Recognition Using Precise Location Detection and ANN for Robot Manipulator," International Conference on Control, Artificial Intelligence, Robotics \& Optimization, 2017.

22. Muknnd Dange, Nitin Afzulpurkar, Supatbra Plermkamon," Adaptive Linear Robot Control for Tracking and Grasping a Dynamic Object," IEEE ICIT'02, 2016.

23. Yun Lin, Shaogang Ren, Matthew Clevenger Yu Sun, "Learning Grasping Force from Demonstration," IEEE International Conference on Robotics and Automation River Centre, May 14-18, 2012.

24. Tuong Phuoc Tho, Nguyen Truong Thinh, "Using ANFIS to Predict Picking Position of the Fruits Sorting System," International Conference on System Science and Engineering (ICSSE), 2017.

25. Kazi Mahmud Hasan, Abdullah -AI-Nahid, K. J. Reza, S. Khatun M. R. Basar, "Sensor-Based Autonomous Colour Line Follower Robot with Obstacle Avoidance," IEEE Business Engineering and Industrial Applications Colloquium (BEIAC) Sensor, 2013.

26. Roshni N, Dr. Sunil Kumar T K, "Pick and Place Robot Using the Centre of Gravity Value of the Moving Object," IEEE Internationa conference on intelligent techniques in control, optimization and signal processing, 2017.

27. Paul Hoffmann, Martin Rudorfer, Jan Guhl, Jorge Kruger," Holo Pick place using V5 Robot", 27th IEEE International Symposium on Robot and Human Interactive Communication (RO-MAN), Aug 2018, pp1

\section{AUTHORS PROFILE}

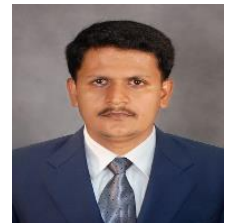

Prof Santhosh S, B.E, MTECH in field of digital electronics and communication. Research Work on Embedded Systems and Robotics.

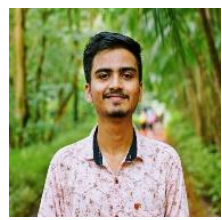

Karthik J $4^{\text {th }}$ Year, B.E (Electronics and Communication Engineering) Finalists at eYRC-2018.

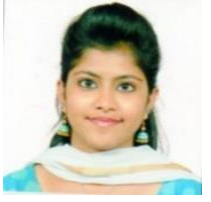

Chesmi B R $4^{\text {th }}$ Year, B.E (Electronics and Communication Engineering) Finalists at eYRC-2018.

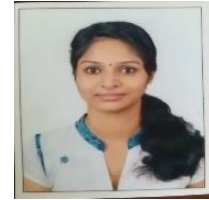

Anju Thomas $4^{\text {th }}$ Year, B.E (Electronics and Communication Engineering) Finalists at eYRC-2018.

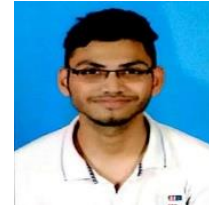

Davis Patel $4^{\text {th }}$ Year, B.E (Electronics and Communication Engineering) Finalists at eYRC-2018. 